\title{
Screening anthelmintic resistance to triclabendazole in Fasciola hepatica isolated from sheep by means of an egg hatch assay

\author{
David Robles-Pérez ${ }^{1}$, José Manuel Martínez-Pérez ${ }^{1}$, Francisco Antonio Rojo-Vázquez ${ }^{1,2}$ \\ and María Martínez-Valladares ${ }^{2^{*}}$
}

\begin{abstract}
Background: In the present study, the detection of anthelmintic resistance to triclabendazole (TCBZ) in sheep infected by Fasciola hepatica was studied using an egg hatch assay (EHA). F. hepatica eggs were recovered from bile and faeces of infected animals by isolates with different grade of anthelmintic resistance to TCBZ: i) a resistant isolate (RT); ii) a susceptible isolate (ST); iii) naturally infected sheep by a susceptible field strain (FST). In the EHA the percentage of hatched eggs were calculated according to the following concentrations of TCBZ diluted in dimethyl-sulfoxide (DMSO): 0.05, 0.2, 1, 5, and $25 \mu \mathrm{g} / \mathrm{ml}$.

Results: In relation to the EHAs carried out with the eggs from bile of sheep infected by ST, differences were found in the percentage of hatched eggs between the control well, only with DMSO, and the two highest concentrations of TCBZ ( 5 and $25 \mu \mathrm{g} / \mathrm{m})(p<0.05)$. However, when we tested the drug with the eggs from the bile of sheep infected by RT, the percentage of hatched eggs was similar among all concentrations. Since the range of hatching varied between isolates, we calculated the ratio of the results of each concentration to its control value confirming the higher hatching in RT than in ST.

We developed an EHA with eggs recovered from faeces in order to avoid the slaughter of sheep. The results of the EHAs with the isolate ST showed differences in the percentage of hatching between the highest concentration $(25 \mathrm{\mu g} / \mathrm{ml})$ and the control well $(p<0.05)$; however, these differences were not confirmed under field conditions with the strain FST.

Conclusions: The ovicidal effect of TCBZ in F. hepatica eggs from bile was shown using a commercial formulation diluted in DMSO with a minimum concentration of $5 \mu \mathrm{g} / \mathrm{ml}$. However, in eggs recovered from faeces the results are not conclusive. The cleaning of eggs recovered from faeces is an important issue that should be reviewed and standardized before comparing results between susceptible and resistant isolates in this kind of EHA.
\end{abstract}

Keywords: Fasciola hepatica, Anthelmintic resistance, Egg hatch assay, Triclabendazole

\section{Background}

Fasciola spp. infects mammals worldwide, mainly ruminants, but also humans can become infected. In ruminants, and especially in sheep, the infection reduces feed conversion, growth, and meat and milk production. Moreover, it is one of the major causes of liver condemnations at abattoirs and interferes with fertility and fecundity [1].

\footnotetext{
* Correspondence: mmarva@eae.csic.es

${ }^{2}$ Instituto de Ganadería de Montaña (CSIC-ULE), Finca de Marzanas, 24346 Grulleros, León, Spain

Full list of author information is available at the end of the article
}

The infection is usually caused, in temperate areas of the world, by the common liver fluke $F$. hepatica. Its prevalence is rising nowadays due to different factors such as climate change, man-made environmental modifications or the presence of anthelmintic resistance (AR) $[1,2]$. AR is the result of repeated administration of the same anthelmintic; moreover, its development has been favoured by ineffective treatment due to the underdosing. AR is the result of repeated treatments of the same anthelmintic although its development is also favoured by the administration of underdosing or overdosing [3].

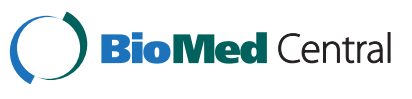

(c) 2015 Robles-Pérez et al. Open Access This article is distributed under the terms of the Creative Commons Attribution 4.0 International License (http://creativecommons.org/licenses/by/4.0/), which permits unrestricted use, distribution, and reproduction in any medium, provided you give appropriate credit to the original author(s) and the source, provide a link to the Creative Commons license, and indicate if changes were made. The Creative Commons Public Domain Dedication waiver (http://creativecommons.org/publicdomain/zero/1.0/) applies to the data made available in this article, unless otherwise stated. 
Currently, the most commonly used drug to control fasciolosis belongs to benzimidazole (BZ) family and is the triclabendazole (TCBZ). TCBZ has been the drug of choice for treating liver fluke infections in livestock for over 20 years [4] since it is the only anthelmintic effective against both $F$. hepatica stages, immature and mature flukes [5]. However, there are several reports describing resistant strains of $F$. hepatica to TCBZ all around the world, in Australia [6], Argentina [7] and also in different European countries [8-11]. Therefore, early detection of resistance is essential, since reversion to susceptibility does not seem to occur [12].

Some in vivo and in vitro tests have been developed to detect the AR in ruminants. Among the in vivo tests, the faecal egg count reduction test (FECRT) is based on the reduction of the number of eggs in faeces after the anthelmintic treatment [13]. Regarding in vitro tests, an egg hatch assay (EHA) has been described to detect BZ resistance in Trichostrongylidae [14, 15]. The EHA is based on the ovicidal properties of some BZs, and on the capacity of eggs from resistant isolates to embrionate and hatch at higher concentrations than those ones from a susceptible isolate [16]. Although the EHA was originally designed to detect AR in gastrointestinal nematodes (GIN), some studies have been carried out with $F$. hepatica eggs from gall bladder and/or faeces using TCBZ, albendazole (ABZ) and their sulphoxide metabolites [17-19].

The aim of this study has been to characterize the susceptibility and resistance of $F$. hepatica isolates to TCBZ by means of an EHA using eggs from gall bladder and faeces.

\section{Methods}

\section{Isolates of $F$. hepatica}

Eight experimentally infected sheep with two $F$. hepatica isolates having different levels of resistance or susceptibility to TBCZ were used. The susceptible isolate to TCBZ (ST) was the Shrewsbury/South Gloucester isolate (Ridgeway Research Ltd Company, UK); the TCBZ-susceptibility of this isolate was confirmed in a clinical trial by Martínez-Valladares et al. [11]. The resistant isolate to TCBZ (RT) was characterized by Álvarez-Sánchez et al. [10] in a flock located in the Spanish province of León; the egg reduction in this flock after the treatment of sheep with TCBZ was $81.8 \%$ on 16 day after treatment and $75.7 \%$ on 30 day after treatment. The molecular characterization of RT was recently described by Martínez-Valladares and Rojo-Vázquez [20].

On the other hand, naturally infected sheep with a TCBZ-susceptible field strain (FST) situated in Palencia, Spain, was also tested. The susceptibility of this strain was previously shown by Robles-Pérez et al. [21].

\section{Egg hatch assays}

A commercial formulation of TCBZ (Fasinex ${ }^{\circ}$ ) diluted in dimethyl sulfoxide (DMSO) was used to carry out the EHAs. The concentration of TCBZ in this commercial formulation was $50 \mathrm{mg} / \mathrm{ml}$. Dilutions of 10, 40, 200, 1000 and $5000 \mu \mathrm{g} / \mathrm{ml}$ were prepared to obtain a final concentration in the wells of $0.05,0.2,1,5$, and $25 \mu \mathrm{g} /$ $\mathrm{ml}$ after adding $10 \mu \mathrm{l}$ of each dilution to a total volume of $2 \mathrm{ml}$. In all EHAs, control wells with $10 \mu \mathrm{l}$ of DMSO were included.

Eggs from faeces were obtained by sedimentation [22] from animals infected by ST and from a pool of faeces of sheep naturally infected by FST. Four sheep, two infected with ST and two with RT, were killed by injection of sodium pentobarbital $\left(\right.$ Dolethal $\left.^{\circ}\right)$ into the jugular vein in order to recover eggs from the bile. F. hepatica eggs were directly recovered from the gall bladder and washed several times with tap water by sedimentation. The slaughter of animals complies with national regulations (R.D. 53/ 2013) and with all animal welfare standards, taking account all necessary moral and ethical issues in the use of experimental animals.

For the EHA, a 24 well cell culture plate was used and all anthelmintic concentrations were tested in duplicate. Into each well, $1890 \mu \mathrm{l}$ of water, $100 \mu \mathrm{l}$ of water with 30-50 eggs, and $10 \mu \mathrm{l}$ of each dilution were placed. Two control wells containing $10 \mu \mathrm{l}$ of DMSO, without TCBZ, were also included. Plates were incubated for 14 days at $25{ }^{\circ} \mathrm{C}$ in darkness. They were then placed under light for $2 \mathrm{~h}$ to stimulate hatching of the miracidia. The number of eggs hatched, embryonated, and unembryonated were counted. All EHA assays were repeated five times for each isolate.

\section{Data and statistical analysis}

The percentage of hatched eggs was calculated for each isolate, using the following formula:

Percentage of hatching $=$ (number of hatched eggs $/$ total number of eggs) $\times 100$

The number of eggs is the sum of hatched, embryonated, and unembryonated eggs (egg in morula stage, without miracidium). The results reported in this study are the mean of five repetitions of each EHA.

With the aim to compare two EHAs with different hatch ranges, a ratio of the results of each concentration to the control was calculated, using the following formula:

$$
\begin{aligned}
& \text { Ratio }=(\% \text { hatching of each concentration } / \\
& \% \text { hatching of control) } \times 100
\end{aligned}
$$

The data were analyzed using the statistical computer package for social sciences SPSS. A one-way ANOVA was used to assess differences. The Dunnett test was carried out to confirm significant differences between concentrations 
and the control group. Differences of less than $5 \%$ were considered significant $(P<0.05)$.

\section{Results}

\section{EHA with eggs from bile}

In the EHAs carried out with the eggs from bile, we compared two isolates, one susceptible (ST) and another resistant (RT) to TCBZ (Fig. 1).

The percentages of hatching in ST isolate are shown in Fig. 1a. The results show that the lower the concentration of the drug is, the higher the percentage of hatched eggs, ranging from 43 to $53 \%$, with a value of $63 \%$ in the control well. The differences between the two highest concentrations ( 5 and $25 \mu \mathrm{g} / \mathrm{ml}$ ) and the control were significant $(p<0.05)$. On the other hand, the percentage of hatched eggs in RT ranged from 19 to $21 \%$, being $22 \%$ in the control well (Fig. 1b). In this isolate, no significant differences were shown between concentrations.

After calculating the ratio of the results of each concentration to its control value, in both isolates (Fig. 2), we confirmed the higher hatching in RT than in ST.

\section{EHA with eggs from faeces}

Figure 3 shows the results of percentage of hatched eggs in the susceptible isolates. The hatching percentage ranged from 17 to $23 \%$, with a control value of $26 \%$ for ST. Significant differences were observed between the highest concentration $(25 \mu \mathrm{g} / \mathrm{ml})$ and the control well $(p<0.05)$ (Fig. 3a).

Using a field strain, FST, also susceptible to TCBZ (Fig. 3b), the percentages of hatching were similar between concentrations, ranging from 19 to $29 \%$, being

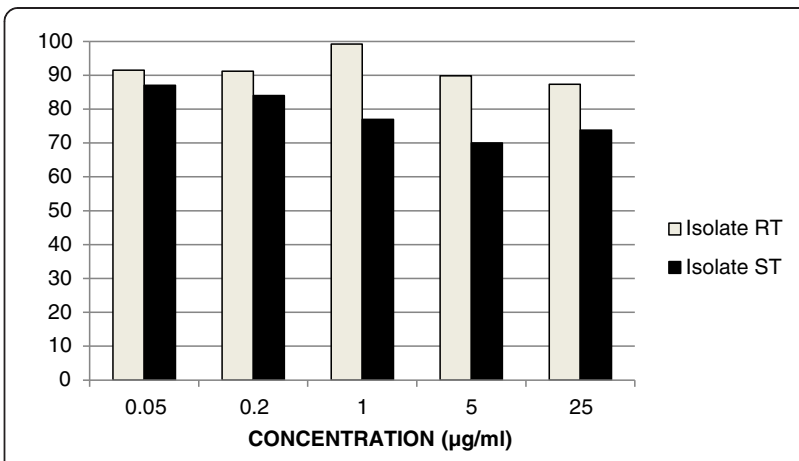

Fig. 2 Ratio of hatched eggs from bile. The ratio for each concentration to its corresponding control for the susceptible and resistant isolates was showed

$33 \%$ in the control well; no significant differences were shown between concentrations.

\section{Discussion}

The study of AR to BZs in ruminants infected by helminth parasites is an important issue to avoid its development and spread. Several authors have used the in vitro technique EHA in order to characterize strains of GIN and $F$. hepatica in sheep flocks $[3,14$, 17-19, 23]. In the present study, the EHA has been adapted and modified to detect the AR to TCBZ in sheep infected by $F$. hepatica.

TCBZ metabolism includes ruminal and hepatic biotransformations in metabolites as TCBZ-sulphoxide, TCBZsulphone, hydroxy-TCBZ, hydroxy-TCBZSO and hydroxy$\mathrm{TCBZSO}_{2}$ [24]. The TCBZ and its sulphoxide and sulphone metabolites contribute to anthelmintic activity and variations in the regional specificity in the levels of disruption to the tegument of the fluke are presents [25]. The drug and

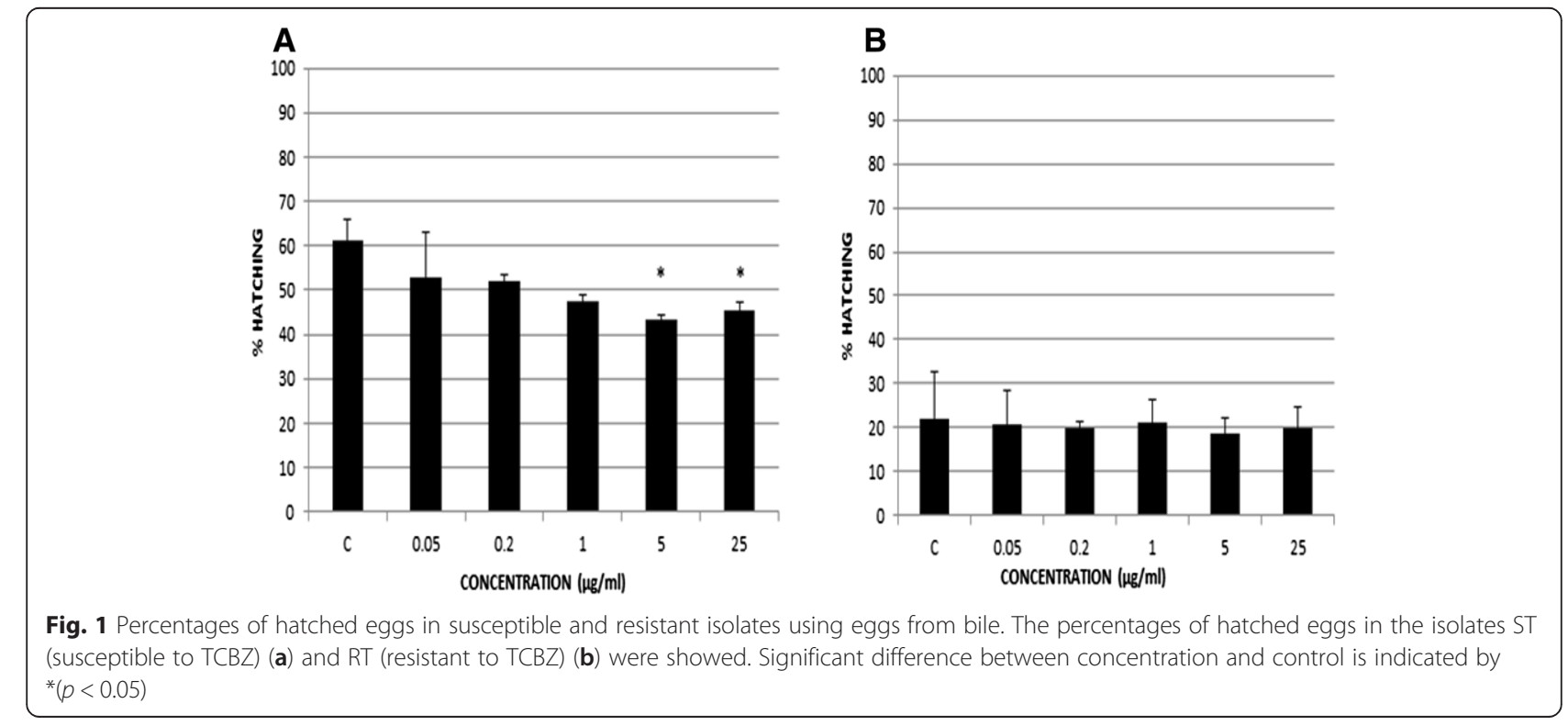



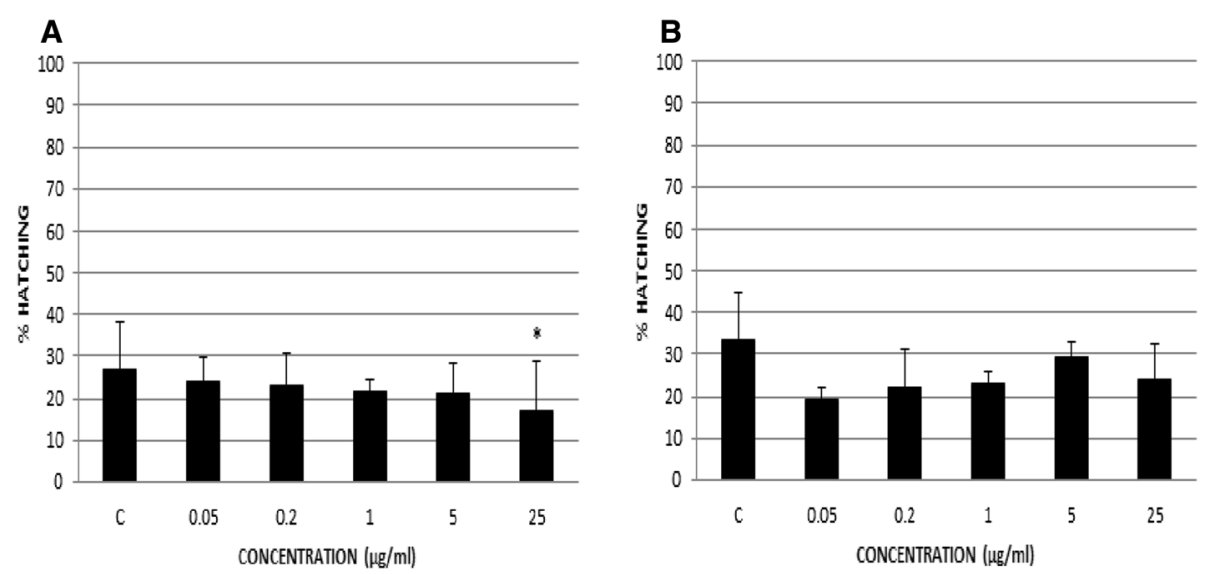

Fig. 3 Percentage of hatched eggs in susceptible isolates using eggs from faeces. The percentages of hatched eggs in the isolates ST (susceptible to TCBZ) (a) and FST (field susceptible strain to TCBZ) (b) were showed. Significant difference between concentration and control is indicated by * $(p<0.05)$

metabolites bind to $\beta$-tubulin which prevents the process of polymerization to form microtubules in the fluke [26]. Accordingly, the integrity of the surface membrane of the parasite is altered leading to damage to the integument and causing the death of the fluke [4].

Firstly, we carried out an EHA using a commercial formulation, Fasinex ${ }^{\circ}$, and eggs from bile. Both, TCBZ and its sulfoxide metabolite were used by Álvarez et al. [17] in a preliminary study testing two formulations of TCBZ in eggs from bile, one diluted with methanol and another one with DMSO. These authors showed a range of hatching of $45-80 \%$ using concentrations of TCBZ between 5 and $20 \mathrm{nmol} / \mathrm{ml}$, equivalent to $1.8-7.2 \mu \mathrm{g} / \mathrm{ml}$; however, they did not find any ovicidal effect of TCBZ in egss from susceptible and resistant strains to this drug. Due to this fact, in the current study we extended the range of concentrations to test the efficacy of TCBZ in eggs from bile, between 0.05 and $25 \mu \mathrm{g} / \mathrm{ml}$. After comparing the percentages of hatching between the susceptible (ST) and resistant (RT) isolates, we found only significant differences between the control and the two highest concentrations (5 and $25 \mu \mathrm{g} / \mathrm{ml})$ in ST $(p<0.05)$, suggesting an ovicidal effect of the drug in this case. However, when the EHA was carried out with eggs from $\mathrm{RT}$, no differences were found among concentrations (Fig. 1b). Recently, Fairweather et al. [18] tested a $60 \mu \mathrm{g} /$ $\mathrm{ml}$ concentration of the sulfoxide metabolite of TCBZ in the same RT isolate that we used in the current study. This author found a very low level of hatching in RT $(<2 \%)$ and therefore they classified the isolate as susceptible. In the present study we showed a percentage of hatching of $20.5 \%$ after testing the highest concentration $(25 \mu \mathrm{g} / \mathrm{ml})$; moreover, no significant differences were described between any concentration and the control using the same RT isolate. The reason of these different results in relation to resistance of RT could be due to the different formulations of TCBZ. Unlike our EHAs, Fairweather et al. [18] used a higher concentration and the sulphoxide metabolite of the drug, not the pure TCBZ.

With the aim to compare the results described between the susceptible and resistant isolates, we calculated a ratio for each isolate and concentration, since the hatching ranges of both isolates were different, probably due to the variability of the technique or the quality of eggs recovered (Fig. 2). The ratios show that the hatching was higher in RT, confirming its higher level of resistance.

On the other hand, with the purpose to avoid the slaughter of sheep to detect the AR using in vitro techniques, we developed an EHA with eggs of $F$. hepatica collected from faeces. With the aim to determine the repeatability of this technique using eggs from faeces, we only tested susceptible isolates, ST and FST. In this case, we observed that the hatching ranges were similar between each other (Fig. 3a and b), however, we only showed significant differences between the highest concentration $(25 \mu \mathrm{g} / \mathrm{ml})$ and the control well in ST. This finding was not confirmed in FST. It is important to note that the percentages of hatching in ST were lower than those obtained in the EHA using eggs from bile. The reason of the low hatching rates could be the presence of rest of faeces or impurities. Indeed, according to Rowcliffe and Ollerenshaw, [27] one of the critical factors for hatching is that the eggs must have become freed from the faeces. Robles-Pérez et al. [19] carried out EHAs to detect the resistance to ABZ using $F$. hepatica eggs recovered from faeces. In that case the percentages of hatching in the control wells were 33, 57 and $71 \%$ for a susceptible strain and $49 \%$ for a resistant strain. Therefore, there is a great variability in the hatching rates. It seems that the methodology to 
recover eggs from faeces is an important issue in the EHA, therefore, this step needs more review and standardization before comparing results between susceptible and resistant isolates.

\section{Conclusions}

The ovicidal effect of TCBZ in F. hepatica eggs from bile was shown using a commercial formulation diluted in DMSO with a minimum concentration of $5 \mu \mathrm{g} / \mathrm{ml}$. We compared the hatching rates of two isolates, one susceptible and another resistant to TCBZ, and we only found significant differences between the two highest concentrations (5 and $25 \mu \mathrm{g} / \mathrm{ml}$ ) and the control well in the susceptible isolate. However, in eggs recovered from faeces the results are not conclusive. Significant differences were shown in the percentages of hatching between the highest concentration $(25 \mu \mathrm{g} / \mathrm{ml})$ and the control in a susceptible isolate, but these results were not confirmed under field conditions with another susceptible strain.

\section{Abbreviations}

TCBZ: Triclabendazole; EHA: Egg hatch assay; RT: Resistant isolate; FST: Susceptible field strain; ST: Susceptible isolate; DMSO: Dimethylsulfoxide; AR: Anthelmintic resistance; FECRT: Faecal egg count reduction test; GIN: Gastrointestinal nematodes; ABZ: Albendazole

\section{Competing interests}

The authors declare that they have no competing interests.

\section{Authors' contributions}

DRP wrote the initial manuscript and performed the experiments. JMMP contributed to the realization of the experiments and the slaughter of animals. FARV and MMV coordinated the experiments and made the revisions required for submission of the final manuscript. All authors read and approved the final manuscript.

\section{Acknowledgements}

This study has been funded by the national project RTA2010-00094-C03-02. The work of María Martínez-Valladares has been supported by a postdoctoral Jae-Doc contract from the Consejo Superior de Investigaciones Científicas (CSIC) and co-funded by the European Social Fund.

\section{Author details}

${ }^{1}$ Faculty of Veterinary Medicine, University of León, Campus de Vegazana, 24071 León, Spain. ${ }^{2}$ Instituto de Ganadería de Montaña (CSIC-ULE), Finca de Marzanas, 24346 Grulleros, León, Spain.

Received: 23 February 2015 Accepted: 18 August 2015 Published online: 28 August 2015

\section{References}

1. Rojo-Vázquez FA, Meana A, Valcárcel F, Martínez-Valladares M. Update on trematode infections in sheep. Vet Parasitol. 2012;30:15-38.

2. Martínez-Valladares M, Robles-Pérez D, Martínez-Pérez JM, Cordero-Pérez C, Famularo MR, Fernández-Pato N, et al. Prevalence of gastrointestinal nematodes and Fasciola hepatica infections in sheep in the northwest of Spain: relation to climatic conditions and/or man-made environmental modifications. Parasit Vectors. 2013;6:282-90

3. FAO (Organización de las Naciones Unidas para la Agricultura y la Alimentación). Resistencia a los antiparasitarios: Estado actual con énfasis en América Latina. Roma, Italia: Dirección de Producción y Sanidad Animal de la $\mathrm{FAO} ; 2003$.

4. Brennan GP, Fairweather I, Trudgett A, Hoey EM, McCoy M, McConville M, et al. Understanding triclabendazole resistance. Exp Mol Pathol. 2007:82:104-9.
5. Boray J, Crowfoot P, Strong M, Allison J, Schellenbaum M, von Orelli M, et al. Treatment of immature and mature Fasciola hepatica infections in sheep with triclabendazole. Vet Rec. 1983;113:315-7.

6. Overend DJ, Bowen FL. Resistance of Fasciola hepatica to triclabendazole. Aust Vet J. 1995;72:275-6.

7. Olaechea F, Lovera V, Larroza M, Raffo F, Cabrera R. Resistance of Fasciola hepatica against triclabenzadole in cattle in Patagonia (Argentina). Vet Parasitol. 2011;178:364-6.

8. Mitchell GBB, Maris L, Bonniwell MA. Triclabendazole-resistant liver fluke in Scottish sheep. Vet Rec. 1998;143:399.

9. Moll L, Gaasenbeek CP, Vellema P, Borgsteede FH. Resistance of Fasciola hepatica against triclabendazole in cattle and sheep in the Netherlands. Vet Parasitol. 2000;91:153-8.

10. Álvarez-Sánchez MA, Mainar-Jaime RC, Pérez-García J, Rojo-Vázquez FA. Resistance of Fasciola hepatica to triclabendazole and albendazole in sheep in Spain. Vet Rec. 2006;159:424-5.

11. Martínez-Valladares M, Famularo MR, Fernández-Pato N, Castañón-Ordóñez L, Cordero-Pérez C, Rojo-Vázquez FA. Efficacy of nitroxynil against Fasciola hepatica resistant to triclabendazole in a naturally infected sheep flock. Parasitol Res. 2010;107:1205-11.

12. Roos MH, Kwa MSG, Grant WN. New genetic and practical implications of selection for anthelmintic resistance in parasitic nematodes. Parasitol Today. 1995;11:148-50.

13. Coles GC, Bauer C, Borgsteede FHM, Geerts S, Dlei TR, Taylor MA, et al. World Association for the Advancement of Veterinay Parasitology (W.A.A.V.P.) methods for the detection of anthelmintic resistance in nematodes of veterinary importance. Vet Parasitol. 1992;44:35-44.

14. Martínez-Valladares M, Famularo MR, Fernández-Pato N, Cordero-Pérez C, Castañón-Ordóñez L, Rojo-Vázquez FA. Characterization of a multidrug resistant Teladorsagia circumcincta isolate from Spain. Parasitol Res. 2012;10:2083-7.

15. Martínez-Valladares M, Martínez-Pérez JM, Robles-Pérez D, Cordero-Pérez C, Famularo MR, Fernández-Pato N, et al. The present status of anthelmintic resistance in gastrointestinal nematode infections of sheep in the northwest of Spain by in vivo and in vitro techniques. Vet Parasitol. 2013;191:177-81.

16. Whitlock HV, Kelly JD, Porter CJ, Griffin DL, Martin ICA. In vitro screening for anthelmintic resistance in strongyles of sheep and horses. Vet Parasitol. 1980;7:215-32

17. Álvarez L, Moreno G, Moreno L, Ceballos L, Shaw L, Fairweather I, et al. Comparative assessment of albendazole and triclabendazole ovicidal activity on Fasciola hepatica eggs. Vet Parasitol. 2009;164:211-6.

18. Fairweather I, MCShane DD, Shaw L, Ellison SE, O'Hagan NT, York EA, et a Development of an egg hatch assay for the diagnosis of triclabendazole resistance in Fasciola hepatica: Proof of concept. Vet Parasitol. 2012;183:249-59.

19. Robles-Pérez D, Martínez-Pérez JM, Rojo-Vázquez F, Martínez-Valladares M. Development of an egg hatch assay for the detection of anthelmintic resistance to albendazole in Fasciola hepatica isolated from sheep. Vet Parasitol. 2014;203:217-21.

20. Martínez-Valladares M, Rojo-Vázquez FA. Intraspecific mitochondrial DNA variation of Fasciola hepatica egss from sheep with different level of anthelmintic resistance. Parasitol Res. 2014;113:2733-41.

21. Robles-Pérez D, Martínez-Pérez JM, Rojo-Vázquez FA, Martínez-Valladares M. The diagnosis of fasciolosis in faeces of sheep by means of a PCR and its application in the detection of anthelmintic resistance in sheep flocks naturally infected. Vet Parasitol. 2013;197:277-82.

22. MAFF (Ministry of Agriculture Fisheries and Food). Manual of Veterinary Parasitological Laboratory Techniques. Technical bulletin N. 18, HMSO, London, 1986.

23. Coles GC, Jackson F, Pomroy WE, Prichard R, von Samson-Himmelstjerna G, Silvestre $A$, et al. The detection of anthelmintic resistance in nematodes of veterinary importance. Vet Parasitol. 2006;136:167-85.

24. Virkel G, Lifschitz A, Sallovitz J, Pis A, Lanusse C. Assessment of the main metabolism pathways for the flukicidal compound tri-clabendazole in sheep. J Vet Pharmacol Ther. 2006;29:213-23.

25. Halferty L, Brennan GP, Trudgett A, Hoey EM, Fairweather I. The relative activity of triclabendazole metabolites against the liver fluke, Fasciola hepatica. Vet Parasitol. 2009;159:126-38.

26. Stitt AW, Fairweather I. Fasciola hepatica: tegumental surface changes in adult and juvenile flukes following treatment in vitro with the sulphoxide metabolite of triclabendazole (Fasinex). Parasitol Res. 1993;79:529-36.

27. Rowcliffe SA, Ollerenshaw CB. Observations on the bionomics of the egg of Fasciola hepatica. Ann trop Med Parasitol. 1960;54:172-81. 